\title{
Discrimination of particulate matter emission sources using stochastic methods
}

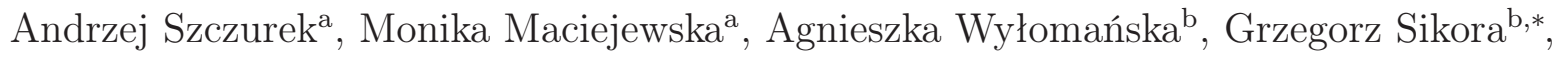 \\ Michał Balcerek ${ }^{\mathrm{b}}$, Marek Teuerle ${ }^{\mathrm{b}}$ \\ ${ }^{a}$ Faculty of Environmental Engineering, Wrocław University of Science and Technology, 27 Wybrzeże \\ Wyspianskiego St., 50-370 Wroctaw, Poland \\ ${ }^{b}$ Faculty of Pure and Applied Mathematics, Wrocław University of Science and Technology, 27 Wybrzeze \\ Wyspiańskiego St., 50-370 Wrocław, Poland
}

\begin{abstract}
Particulate matter (PM) is one of the criteria pollutants which has been determined as harmful to public health and the environment. For this reason the ability to recognize its emission sources is very important. There are a number of measurement methods which allow to characterize PM in terms of concentration, particles size distribution, and chemical composition. All these information are useful to establish a link between the dust found in the air, its emission sources and influence on human as well as the environment. However, the methods are typically quite sophisticated and not applicable outside laboratories. In this work, we considered PM emission source discrimination method which is based on continuous measurements of PM concentration with a relatively cheap instrument and stochastic analysis of the obtained data. The stochastic analysis is focused on the temporal variation of PM concentration and it involves two steps: 1) recognition of the category of distribution for the data i.e. stable or the domain of attraction of stable distribution and 2) finding best matching distribution out of Gaussian, stable and normal-inverse Gaussian (NIG). We examined six PM emission sources. They were associated with material processing in industrial environment, namely machining and welding aluminum, forged carbon steel and plastic with various tools. As shown by the obtained results, PM emission sources may be distinguished based on statistical distribution of PM concentration variations. Major factor responsible for the differences detectable with our method, was the type of material processing and the tool applied. In case different materials were processed by the same tool the distinction of emission sources was difficult. For successful discrimination it was crucial to consider size-segregated mass fraction concentrations. In our opinion the presented approach is very promising. It deserves further study and development.
\end{abstract}

Keywords: PM, emission source, distribution fitting, stable domain of attraction

\footnotetext{
The research was financed by National Science Foundation Poland under contract number UMO2012/07/B/ST8/03031

*Corresponding author

Email addresses: andrzej.szczurek@pwr.edu.pl (Andrzej Szczurek),
}

Preprint submitted to Physica A: Statistical Mechanics and its Applications

April 25, 2016 


\section{Introduction}

Particulate matter (PM) is a general term used for a mixture of solid particles and liquid droplets in the air [1]. These suspended particles vary in size, composition and origin.

PM is one of the criteria pollutants that has been determined to be harmful to public health and the environment. The adverse effects of inhaling dust depend strongly on the chemical composition of particles as well as their specific parameters such as shape, diameter, size, morphology, surface area, structure, electric charge, solubility. The chemical and physical properties of PM result mainly from its source, mechanism of formation, emission process, distance from the source. Therefore, knowledge about these factors is important for the characterization of dust behaviour in air and possible health risks [2, 3].

Identifying major pollution sources that contribute to concentration of PM is essential for developing an effective air quality management plan. In practice, there are many methods of deriving information about sources of PM emission. The measurement approach is the most reliable. It involves sampling and laboratory analyses to identify chemical composition and physical properties of collected particles. Both chemical composition and size can provide valuable insights into the source of dust particles. Identifying the composition of the particulate can trace back to the source of pollution.

The characterization of a particular process emissivity must be done using high sensitivity methods. There are several of them. Electron microscopy yields information about particles' shape and diameter. This technique is important for characterization of surface structure and aspherical determination of coarser particles. Computer-controlled electron microscopy is useful for counting, sizing, distribution, and morphology of finer particles. Energy Dispersive X-ray Spectroscopy allows to determine elemental chemical composition. X-ray Fluorescence [4] and Inductively Coupled Plasma Mass Spectroscopy [5] provide information about elemental concentrations. The Scanning Mobility Particle Sizer (SMPS) is one of the most commonly used instruments to measure the aerosol spectra in the size range from 2.5 to $1000 \mathrm{~nm}$ [5]. Cascade impactors are used to classify particles according to their size on the substrates placed on different stages of the device [6]. The size of particles in the ultrafine/nano size region is measured using a differential mobility analyser [7] in the SMPS. The most sophisticated version is capable of counting particles between $1 \mathrm{~nm}$ to $1000 \mathrm{~nm}$ in diameter. A Condensation Nucleus Counter [8] can count ultrafine and nano aerosols by a process that involves the condensation of a vapour (usually 1-butanol, 1-propanol or water) onto the surface of the constituent particles to make them large enough to be detected by optical light scattering. Electrical charge measurement can be used to measure the surface area of a particle directly and the diffusion charging type of instrument can be used to measure the active or Fuchs surface area [9].

In contrast to instruments that measure ultrafine particle concentration or number, there are not many instruments available that can be considered as suitable to measure the mass

monika.maciejewska@pwr.edu.pl (Monika Maciejewska), agnieszka.wylomanska@pwr.edu.pl (Agnieszka Wyłomańska), grzegorz.sikora@pwr.edu.pl (Grzegorz Sikora), michal.balcerek@pwr.edu.pl (Michał Balcerek), marek.teuerle@pwr.edu.pl (Marek Teuerle ) 
of ultrafine aerosols in real time. Of those that are available there is only one instrument potentially sensitive enough and that can be realistically and conveniently used to perform such measurements. This instrument is based on the principle of a tapered element oscillating microbalance $[10,11]$.

Knowledge about particles' shape, structure, diameter and composition obtained by the analysis of individual particles with an electron microscopy and different spectroscopic methods yield important information for accurate source identification. However, these techniques are expensive, time-consuming and require specialised professional qualifications. Hence, their application field is limited.

The aim of this study is a method that allows to discriminate dust emission sources using relatively simple measurements and stochastic methods of data analysis. We focused attention on this approach, because it is relatively rapid and cost-effective in application.

Our work is based on the assumption that characteristic features of dust sources are reflected in the variability of a PM concentration. This assumption is reasonable, because distribution of emitted aerosol depends on dynamic properties of dust particles which are strongly affected by different phenomena occurring during their formation.

PM may be emitted from a variety of sources. In this study we focused on machining $[12,13,14]$ and industrial processes involving heat, such as welding, soldering, because each of them may be accompanied by a high dust emission [15].

Machining is a manufacturing process in which a cutting tool removes excess material from a workpiece to produce the desired shape. It was proved that metal machining processes produce broad spectra of particles. Most of the dusts generated during machining consist of very fine particles (diameter smaller than $1 \mu \mathrm{m}$ ). Their numeric concentration is around 10 to 35 times greater than that of the biggest particles (around $5 \mu \mathrm{m}$ in diameter) and depends on the type of cutting tool used [16]. All machining processes generate aerosols (dry or wet)[17], which can be harmful to health of machine operators and the environment [18]. For the metallic dust emission during machining processes, the following process parameters were found important: cutting conditions (cutting speed, depth of cut and feed rate), tool geometry (rake angle, and lead angle) and workpiece material. These parameters influence the shearing of metal, the friction and the plastic deformation. Friction can thus cause particle detachment by various means [19].

Welding is a process involving heat. This category of processes are largely a source of aerosols containing very small particles in the $1 \mathrm{~nm}$ to $100 \mathrm{~nm}$ size range. They are formed from gases through transient nucleation via the hot vapour condensation, primary particle formation, coagulation and aggregation route. Nucleation processes accompany the emissions from high temperature sources. The efficiency of both coagulation and condensation decreases as particle size increases. Therefore particles do not grow by these processes beyond approximately $1 \mu \mathrm{m}$. Particles greater than about $1 \mu \mathrm{m}$ are typically generated mechanically by the break-up of larger solid particles. The amount of energy required to break these particles into smaller increases as the size decreases, which effectively establishes a lower limit for the production of these coarse particles of approximately $1 \mu \mathrm{m}$. Temperature is especially important in welding processes that are associated with the emission of welding fume - the mixture of dust and gases, the source of which is high temperature and the radiation of 
welding arc. Welding fume particles range in size from 0.005 to $20 \mu \mathrm{m}$, although less than $10-30 \%$ (depending on the welding process) of the fume mass is larger than $1 \mu \mathrm{m}$ [15].

The structure of the paper is as follows. In section 2 we provide the details of experimental part of our work, associated with PM concentration measurements in the vicinity of various emission sources. In section 3 we present the methodology of the stochastic data analysis. In particular, the method of recognizing a category of data distribution is given in section 3.1. Details of fitted distributions are provided in section 3.1 and 3.2. Statistical tests applied to determine the quality of fit are introduced in section 3.2. In section 4 we present results of stochastic analysis individually for each investigated PM emission source. The obtained results are discussed and summarized in section 5 .

\section{Experimental}

In this work we investigated several PM emission sources, which were associated with material processing in industrial environment. The essential elements of emission sources description are presented in Table 1. They include: processed material, type of processing and the tool/machine applied. There were considered emissions resulting from machining and welding of aluminum, forged carbon steel, plastic and cast iron. The processing was realized by means of various specialized tools/machines. All emission sources were located indoors in one industrial hall.

The discrimination of emission sources was based on the measurement data. It was col-

Table 1: Description of the investigated PM emission sources.

\begin{tabular}{|c|c|c|c|}
\hline Source ID & material & process & tool \\
\hline $\mathrm{Al}, \mathrm{T} 1$ & aluminum $(\mathrm{Al})$ & machining & Hass machining machine (T1) \\
\hline $\mathrm{Al}, \mathrm{T} 2$ & aluminum $(\mathrm{Al})$ & machining & Monoblock machining machine (T2) \\
\hline FC,T3 & forged carbon steel $(\mathrm{FC})$ & machining & Universal machining machine (T3) \\
\hline FC,T4 & forged carbon steel $(\mathrm{FC})$ & welding & Ordinary welding (T4) \\
\hline PL,T2 & plastic $(\mathrm{PL})$ & machining & Monoblock machining machine (T2) \\
\hline $\mathrm{Al}, \mathrm{T} 5$ & aluminum $(\mathrm{Al})$ & welding & Telwin welder superior (T5) \\
\hline
\end{tabular}

lected in course of PM concentration monitoring in the vicinity of the examined sources. Each of them was monitored individually for a period of one day. Measurements were performed in a continuous manner. The data was recorded in the form of the time series of measured values with the temporal resolution of $10 \mathrm{~s}$. For the analysis we considered first 800 observations of the emission source operation, which corresponds to about $2 \mathrm{~h}$.

We applied the measuring instrument which utilised light-scattering laser photometery. It allowed to simultaneously measure size-segregated mass fraction concentrations corresponding to PM1, PM2.5, PM4, PM10 and Total PM size fractions. PM1 stands for particles with diameter up to $1 \mu \mathrm{m}$. The PM properties are usually expressed in terms of the aerodynamic diameter. Taking into consideration this parameter, PM is divided on two fraction: coarse (a size ranging from 2.5 to $10 \mu \mathrm{m}$ ) and fine (a size up to $2.5 \mu \mathrm{m}$ ). The particles in the last fraction which are smaller than $0.1 \mu \mathrm{m}$ are classified as ultrafine PM. In the analysis 
presented in this work we focused on small coarse and fine particles. More specifically, there were considered three ranges of particles size: $\mathrm{f} 1 \equiv(0,1] \mu \mathrm{m}, \mathrm{f} 2 \equiv(1,2.5] \mu \mathrm{m}, \mathrm{f} 3 \equiv(2.5,4] \mu \mathrm{m}$. In order to obtain the data on PM concentration in those ranges, the raw measurement data was transformed by applying subtraction operation accordingly, eg. f2=PM2.5-PM1. Moreover, as we were interested in temporal variation of PM concentration, the direct input to our analysis were the differenced time series of PM concentration referring to fractions f1, f2 and f3 i.e. the time series of concentration increments.

\section{Methodology}

In this section we present the methodology that was used for data analysis. We concentrate on the recognition of distribution of appropriate datasets corresponding to concentration of dust in the air and distinction of those distributions for different sources of dust emission. The proposed procedure consists of two steps. In the first step we specify the distribution category expressed in the language of the domain of attraction of the distribution corresponding to analysed time series, while in the second step we try to fit the specific distribution that in best way describes the behavior of time series. In the preliminary analysis (step one) we use the graphical test that was introduced in order to recognize if analysed dataset belongs to domain of attraction of Gaussian or strictly stable distribution [26]. Here we can not answer the question which distribution is the best one for analysed data, we only specify category of distribution (fat or heavy tailed). Then, we step forward (second step of the analysis) and by using statistical methods we fit the appropriate distribution to given data. Here we concentrate on three distributions: Gaussian, stable and normal inverse Gaussian (NIG).

\subsection{Recognition of the domain of attraction}

The first step of our analysis is based on modeling of data by using stable distribution approach. More precisely, the analysed data are treated as samples of independent identically distributed (i.i.d.) random variables and we assume the data come from stable distribution, or from the distribution from the domain of attraction of stable distribution. Stable distributions $[20,21]$ belong to a four-parameters class of continuous probability distributions and are denoted $S_{\alpha}(\sigma, \beta, \mu)$. The parameters that specify a stable distribution are: stability parameter $\alpha$, skewness parameter $\beta$, scale and location parameters $\sigma$ and $\mu$, respectively. Stable distribution is a widely-used class of probability distributions mainly for modeling financial data, but also in economics, physics and other applications [22]. They are important because of the generalization of the Central Limit Theorem, which states that stable distributions are the only possible limit (in distribution) of normalized and centered sums of independent and identically distributed random variables. Furthermore, stable distributions can accommodate the fat tails and asymmetry and thus they can fit very well to empirical data.

There are many equivalent definitions of stable random variables. In this section, there are 
stated two of them, i.e. the original definition related to their basic property (Definition 1) and the definition using characteristic function (Definition 2).

Definition 1. [23] Let $X_{1}$ and $X_{2}$ be independent copies of $X$, $a$ and $b$ are any positive constants. A random variable $X$ is stable if and only if

$$
a X_{1}+b X_{2} \stackrel{d}{=} c X+d
$$

for some constants $d \in \mathbb{R}$ and $c \in(0, \infty)$. $X$ is said to be strictly stable $\Longleftrightarrow d=0$. Additionally, constant $c=\left(a^{\alpha}+b^{\alpha}\right)^{1 / \alpha}$ for some $\alpha \in(0,2]$.

Definition 2. [23] Random variable $X$ has a stable distribution, denoted as $S_{\alpha}(\sigma, \beta, \mu)$, if the characteristic function of $X$ has the following form

$$
\Phi_{X}(u)= \begin{cases}\exp \left[-\sigma^{\alpha}|u|^{\alpha}\left(1-i \beta \operatorname{sign}(u) \tan \frac{\pi \alpha}{2}\right)+i \mu u\right], & \alpha \neq 1, \\ \exp \left[-\sigma|u|\left(1+i \beta \operatorname{sign}(u) \frac{2}{\pi} \ln |u|\right)+i \mu u\right], & \alpha=1,\end{cases}
$$

where $\alpha \in(0,2), \beta \in[-1,1], \sigma>0, \mu \in \mathbb{R}$.

Stable distribution does not have a general closed form expression for the density and distribution function, except of three cases with specified parameters $\alpha$ and $\beta$. Namely, Gaussian $\left(S_{2}(\sigma, 0, \mu)=N\left(\mu, 2 \sigma^{2}\right)\right)$, Cauchy $\left(S_{1}(\sigma, 0, \mu)\right)$ and Lévy $\left(S_{\frac{1}{2}}(\sigma, 1, \mu)\right)$ distributions are representations of stable distributions with densities that can be written analytically.

Stable distributions with $\alpha<2$ (that is all except normal distribution) have the so-called heavy tails that behaves like power functions [24], i.e. for $Y \sim S_{\alpha}(\sigma, \beta, \mu)$, where $\alpha<2$, there is satisfied at least one of properties:

$$
\begin{aligned}
& P(Y \geq x) \sim x^{-\alpha} \\
& P(Y \leq x) \sim x^{-\alpha}
\end{aligned}
$$

as $x \rightarrow \infty$. For $\alpha<2$, stable distributions satisfy one of the properties above (when $\alpha<1$, $\beta= \pm 1$ ) and both for all other cases. The 'heavy tails' property indicates that not all moments exist. Namely, for $Y$ with stable distribution $S_{\alpha}(\sigma, \beta, \mu)$ and $\alpha<2$ following condition holds

$$
E|Y|^{p}<\infty \Leftrightarrow 0<p<\alpha
$$

where $p$ is any real number. In particular, the $\operatorname{Var} X=\infty$ and $E Y$ exists only for $\alpha>1$. In the procedure proposed in this paper we take under consideration another important property of stable distributions. Due to the Generalized Central Limit Theorem they attract distributions of sums of random variables with diverging variance, similarly to the Gaussian law that attracts distributions with finite variance [25]. Thus, as the Gaussian law, the stable 
laws naturally appear when evolution of a system or result of an experiment are determined by a sum of random factors.

In this paper in order to recognize the category of distribution for given time series we propose to use the visual test proposed in [26]. The main idea of this algorithm is to distinguish between the domains of attraction of different underlying distributions. The classic result of probability theory states that a normalized sum of arbitrary i.i.d. random variables converges, if at all, to an $\alpha$-stable $(\alpha<=2)$ random variable [27]. The convergence holds in distribution and its rate varies from distribution to distribution [28]. We apply this fact in the following testing procedure [26]:

1. We divide the dataset into non-overlapping consecutive blocks of length $k=1,2, \ldots, 10$. More precisely, for the dataset $x_{1}, x_{2}, \ldots, x_{N}$ the first block contains observations $x_{1}, \ldots x_{k}$, the second $-x_{k+1}, \ldots x_{2 k}$ etc. Next, we sum the values within each block and obtain aggregated data of length $[N / k](k=1$ refers the whole dataset). Finally, we estimate the index of stability $\alpha$ for the constructed data via the regression method $[29,30,31]$.

2. We plot the estimated index of stability with respect to $k=1,2, \ldots, 10$.

(a) If the estimated values converge to 2, then the data are light-tailed and belong to the domain of attraction of the Gaussian law. In particular, if the data are Gaussian, the estimated values should be equal to 2 for most of the cases.

(b) If the estimated values converge to $\alpha<2$, then the data are heavy-tailed and belong to the domain of attraction of the non-Gaussian stable law. In particular, if the data are $(\alpha<2)$-stable, the estimated values should be always close to $\alpha$.

One can enhance this procedure by calculating boxplots for the estimated values $\alpha$. This is intended to help to access if the differences in convergence are statistically justified. The box plot provides a statistical information about the distribution of the values [32]. Precisely, it produces a box and whisker plot for each value of $\alpha$. The box has lines at the lower quartile, median, and upper quartile values. The whiskers are lines extending from each end of the box to show the extent of the rest of the data. Points are drawn as outliers if they are larger than $Q 3+1.5(Q 3-Q 1)$ or smaller than $Q 1-1.5(Q 3-Q 1)$, where $Q 1$ and Q3 are lower and upper quartiles, respectively. This corresponds to the $99.3 \%$ coverage if the data are normally distributed. The plotted whisker extends to the adjacent value, which is the most extreme data value that is not an outlier.

But, how to create boxplots from a single dataset? The idea is to generate more samples from one sample (from its empirical distribution function). This procedure is called bootstrapping in statistics [33].

\subsection{Distribution fitting}

The important challenge in the empirical data modelling is the distribution fitting. After specification of the category of distribution (step one) we step forward and try to fit an appropriate distribution to data. In this part we present various statistical tests based on empirical distribution function that can be used in the distribution fitting. In those testing procedures different statistics measure the vertical distance between the empirical $F_{N}(x)$ 
and theoretical $F(x)$ cumulative distribution function. The lower value of the test statistics means the better fitting of the proposed distribution. Statistical tests serve tools for making quantitative decisions about a sample distribution. They can provide enough statistical evidence to reject a conjecture or hypothesis about the underlying distribution.

In this paper we consider two classes of such tests, namely the Kolmogorov-Smirnov and Cramér-von Mises. The Kolmogoro-Smirnov statistic is given by:

$$
D=\sup _{x}\left\{\left|F_{N}(x)-F(x)\right|\right\} .
$$

It can be written as a maximum of two non-negative supremum statistics:

$$
D^{+}=\sup _{x}\left\{F_{N}(x)-F(x)\right\} \quad \text { and } \quad D^{-}=\sup _{x}\left\{F(x)-F_{N}(x)\right\} .
$$

We also use the Kuiper statistics from the same class, given by

$$
V=D^{+}+D^{-} .
$$

The second class of vertical measures is the Cramér-von Mises family

$$
C M=N \int_{-\infty}^{\infty}\left(F_{N}(x)-F(x)\right)^{2} \psi(x) d F(x) .
$$

The function $\psi(x)$ is a special weight function. For $\psi(x)=1$ we obtain the $W^{2}$ Cramér-von Mises statistic and for $\psi(x)=[F(x)(1-F(x))]^{-1}$ we get $A^{2}$ Anderson and Darling one.

In this paper we consider three possible probability laws underlying the series: Gaussian, stable, and NIG. The stable distribution was presented in section 2 with the Gaussian case with $\alpha=2$.

The NIG distribution, introduced in [34], was chosen as it is able to model symmetric and asymmetric distributions with possibly long tails in both directions. Its tail behavior is often classified as 'semi-heavy', i.e. the tails are lighter than those of stable laws, but much heavier than the Gaussian. Moreover, empirical experience shows an excellent fit of the NIG law to different data [31]. The NIG distribution is characterized by 4 parameters: location parameter $\mu$, tail heaviness $\alpha$, asymmetry parameter $\beta$, and the scale parameter $\delta$. Each of these parameters can have real values and the support of the distribution is the whole real line. The density of NIG distribution with such parameters has the form

$$
f(x)=\frac{\alpha \delta K_{1}\left(\alpha \sqrt{\delta^{2}+(x-\mu)^{2}}\right)}{\pi \sqrt{\delta^{2}+(x-\mu)^{2}}} e^{\delta \gamma+\beta(x-m u)},
$$

where $\gamma=\sqrt{\alpha^{2}-\beta^{2}}$, and $K_{1}$ denotes a modified Bessel function of the third kind [35]. Also the moment generating function has the simple exponential form

$$
M(t)=e^{\mu t}+\delta\left(\gamma-\sqrt{\alpha^{2}-(\beta+t)^{2}}\right) .
$$

Thanks to the simple form of moment generationg function all NIG moments are finite and easily expressible. 

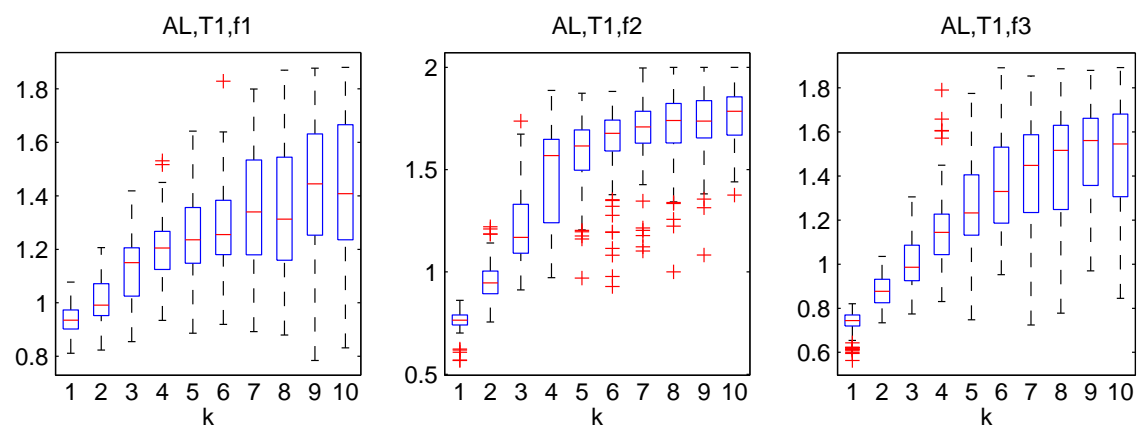

Figure 1: Emission source AL,T1 for three PM fractions f1, f2 and f3; Time series of PM concentration increments (top panels) and the corresponding results of the visual test (bottom panels).

\section{Data analysis results}

\subsection{Emission source $A L, T 1$.}

In this section we consider the statistical description of concentration increments for the PM emitted from aluminum machining using Hass machine. This source is indicated by the symbol: Al,T1.

In Fig. 1 (top panels) we display the differenced time series of PM concentrations recorded in the vicinity of AL,T1 emission source for three PM fractions $\mathrm{f} 1$, $\mathrm{f} 2$ and $\mathrm{f} 3$. In Fig. 1 (bottom panel) there are presented the corresponding results of the visual test, which allows to recognize domain of attraction for the distribution of these data sets. The summary of recognition is given in Table 2. We distinguished stable distribution from the domain of attraction of stable distribution (DOAS) and provided the value of $\alpha$ in each case.

Based on results of visual test, the distributions describing variation of concentration of PM emitted from source AL,T1 belong to the domain of attraction of stable distribution. There are differences between PM fractions, which are reflected in values of $\alpha$ parameter.

In Table 3 there are shown the results of statistical tests regarding a distribution fitting for PM fractions f1, f2 and f3 emitted from source AL,T1. There were considered Gaussian, stable and normal-inverse Gaussian distributions. We present the values of all test statistics applied $D, V, W^{2}$ and $A^{2}$.

The results of distribution fitting to the data representing temporal variation of concentration of PM emitted from source AL,T1 are not obvious. As shown in Table 3 the fraction 


\begin{tabular}{c|c|c|c|} 
& $\mathrm{f} 1$ & $\mathrm{f} 2$ & $\mathrm{f} 3$ \\
\hline \hline AL,T1 & DOAS $\alpha=1.4$ & DOAS $\alpha=1.8$ & DOAS $\alpha=1.6$ \\
\hline AL,T2 & DOAS $\alpha=1.95$ & Stable with $\alpha=1.45$ & Stable with $\alpha=1.3$ \\
\hline FC,T3 & DOAS $\alpha=1.25$ & Stable with $\alpha=0.75$ & DOAS $\alpha=0.8$ \\
\hline FC,T4 & DOAS $\alpha=2$ & DOAS $\alpha=2$ & DOAS $\alpha=2$ \\
\hline PL,T2 & DOAS $\alpha=1.6$ & Stable with $\alpha=1.8$ & Stable with $\alpha=1.6$ \\
\hline AL,T5 & DOAS $\alpha=1.95$ & DOAS $\alpha=1.95$ & DOAS $\alpha=1.95$ \\
\hline \hline
\end{tabular}

Table 2: The results of the visual test applied to determine the the category of distribution for the individual PM fractions emitted from the investigated emission sources.

\begin{tabular}{c|ccc|ccc|ccc}
\multirow{2}{*}{ Test statistic } & \multicolumn{3}{|c|}{$\mathrm{f1}$} & \multicolumn{3}{c|}{$\mathrm{f2}$} & \multicolumn{3}{c}{$\mathrm{f} 3$} \\
\cline { 2 - 10 } & Gaus. & Stab. & NIG & Gaus. & Stab. & NIG & Gaus. & Stab. & NIG \\
\hline$D$ & 0.23 & 0.02 & 0.02 & 0.20 & 0.03 & 0.05 & 0.24 & 0.04 & 0.06 \\
$V$ & 0.45 & 0.03 & 0.03 & 0.39 & 0.06 & 0.08 & 0.47 & 0.07 & 0.08 \\
$W^{2}$ & 18.36 & 0.04 & 0.06 & 12.97 & 0.10 & 0.34 & 18.93 & 0.26 & 0.39 \\
$A^{2}$ & 92.82 & 0.56 & 0.49 & 63.44 & 1.88 & 2.65 & 92.47 & 2.55 & 2.98 \\
\hline
\end{tabular}

Table 3: The results of the statistical tests related to the distribution fitting for the emission source AL,T1. The grey shaded cells corresponds the lowest value of test statistic computed for three considered distributions.

f1 could come from either stable or NIG (according to Table 2 it comes from domain of attraction of stable law). The fractions $\mathrm{f} 2$ and $\mathrm{f} 3$ are suspected to be stable (according to Table 2 they come from domain of attraction of stable law), but the values of test statistics are very close to the case of NIG distribution. To have more information about the distribution of the analysed data we present the plot of the right empirical tail with fitted tails of proposed distribution in Fig. 2. From Fig. 2 we conclude that the results from Tables 2 and 3 are consistent. All three series corresponding to three fractions seem to come from NIG distribution. This is in full accordance with the previous Table 2. The NIG tails proposed for these series are clearly superior and well fitted, see Fig. 2. The parameters of fitted NIG distribution we present in Table 4.

\begin{tabular}{c|c|c|c|} 
& $\mathrm{f} 1$ & $\mathrm{f} 2$ & $\mathrm{f} 3$ \\
\hline \hline AL,T1 & NIG: $\mu=0, \alpha=0.65$, & NIG: $\mu=0, \alpha=1.98$, & NIG: $\mu=0, \alpha=1.11$, \\
& $\beta=0.04, \sigma=0.03$ & $\beta=0, \sigma=0.01$ & $\beta=0, \sigma=0.01$ \\
\hline AL,T2 & NIG: $\mu=0, \alpha=38.37$, & Stable: $\mu=0, \alpha=1.45$, & Stable: $\mu=-0.001, \alpha=1.31$, \\
& $\beta=-0.19, \sigma=0$ & $\beta=0.01, \sigma=0.001$ & $\beta=-0.12, \sigma=0.005$ \\
\hline FC,T3 & NIG: $\mu=0, \alpha=0.11$, & Stable: $\mu=0, \alpha=0.75$, & NIG: $\mu=0, \alpha=0.77$, \\
& $\beta=0.004, \sigma=0.01$ & $\beta=-0.05, \sigma=0.001$ & $\beta=0.01, \sigma=0.002$ \\
\hline FC,T4 & Gauss: $\mu=0, \sigma=0.011$, & Gauss: $\mu=0, \alpha=0.001$, & Gauss: $\mu=0, \alpha=0.013$, \\
\hline PL,T2 & NIG: $\mu=0, \alpha=42.45$, & Stable: $\mu=0, \alpha=1.56$, & Stable: $\mu=0, \alpha=1.18$, \\
& $\beta=-0.22, \sigma=0.002$ & $\beta=-0.22, \sigma=0.001$ & $\beta=-0.025, \sigma=0.005$ \\
\hline AL,T5 & not identified & not identified & not identified \\
\hline \hline
\end{tabular}

Table 4: The parameters of the proposed fitted distributions.

In summary, the statistical description of concentration increments for the PM emitted from aluminum machining using Hass machine (AL,T1) was following:

- fraction f1 - DOAS $\alpha=1.4$, NIG: $\mu=0, \alpha=0.65, \beta=0.04, \sigma=0.03$; 

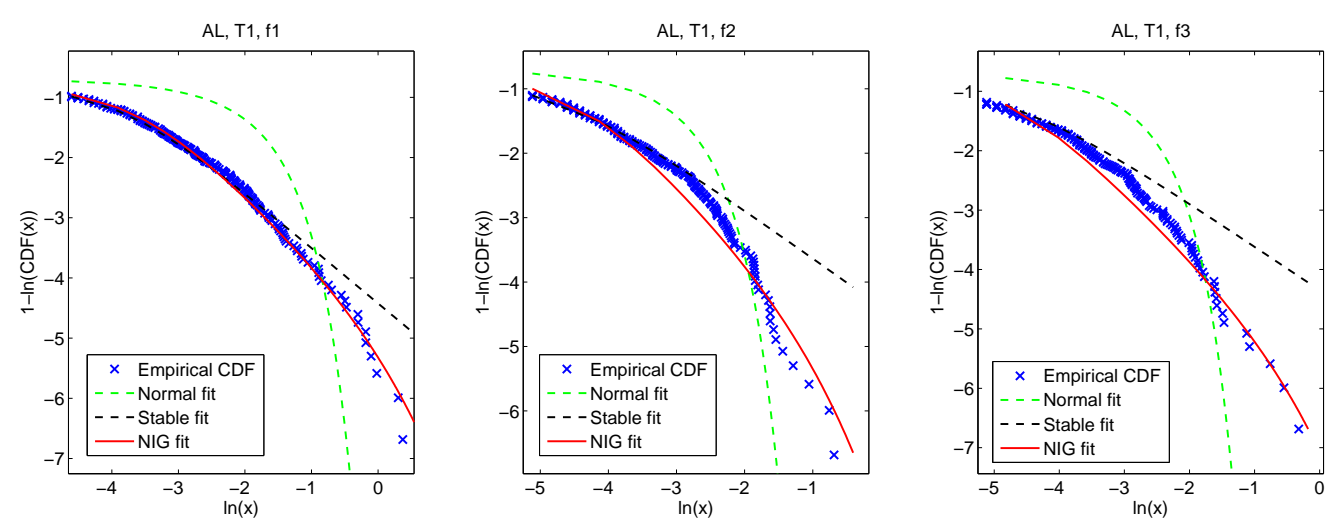

Figure 2: The empirical right tails and fitted tails corresponding the proposed distributions for emission source AL,T1.

- fraction f2 - DOAS $\alpha=1.8$, NIG: $\mu=0, \alpha=1.98, \beta=0, \sigma=0.01$;

- fraction f3 - DOAS $\alpha=1.6$, NIG: $\mu=0, \alpha=1.11, \beta=0, \sigma=0.01$.

For each PM fraction we quoted the identified category of distribution and the proposed fitted distribution with its parameters.

\subsection{Emission source AL,T2.}

In this section we consider the statistical description of concentration increments for the PM emitted from aluminum machining using Monoblock machine. This source is indicated by the symbol: Al,T2.

In Fig. 3 (top panels) we display the differenced time series of PM concentrations recorded in the vicinity of AL,T2 emission source for three PM fractions $\mathrm{f} 1, \mathrm{f} 2$ and $\mathrm{f} 3$. In Fig. 3 (bottom panel) there are presented the corresponding results of the visual test, which allows to recognize domain of attraction for the distribution in case of each PM fraction. The summary of recognition is given in Table 2 .

Based on results of visual test, the distribution describing variation of concentration of PM fraction 11 emitted from source AL,T1 belongs to the domain of attraction of stable distribution. In case of fractions $\mathrm{f} 2$ and $\mathrm{f} 3$, distributions follow stable law. Moreover, $\alpha$ parameter is very similar in both cases.

In Table 5 there are shown the results of statistical tests regarding a particular distribution fitting for PM fractions $\mathrm{f} 1, \mathrm{f} 2$ and $\mathrm{f} 3$ emitted from source AL,T2. We present the values of all test statistics applied. The results of fitting are consistent with Table 2, i.e. the data corresponding the dust fraction $\mathrm{f} 1$ are suspected to come from NIG distribution from the domain of attraction of stable law, the fractions $\mathrm{f} 2$ and $\mathrm{f} 3$ are suspected to be stable. The parameters of fitted distributions we present in Table 4.

In summary, the statistical description of concentration increments for the PM emitted from aluminum machining using Monoblock machine (AL,T2) was following: 

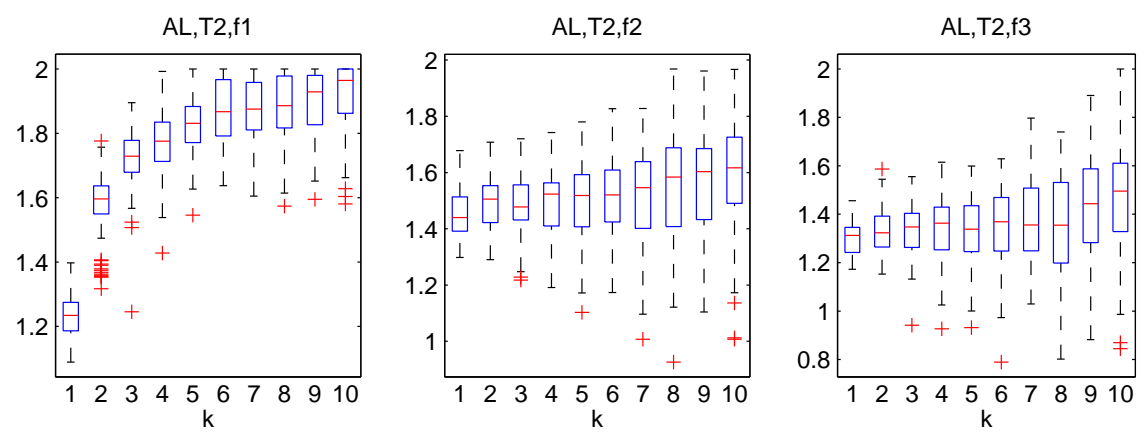

Figure 3: Emission source AL,T2 for three PM fractions f1, f2 and f3; Time series of PM concentration increments (top panels) and the corresponding results of the visual test (bottom panels).

\begin{tabular}{c|ccc|ccc|ccc}
\multirow{2}{*}{ Test statistic } & \multicolumn{3}{|c|}{$\mathrm{f1}$} & \multicolumn{3}{c|}{$\mathrm{f} 2$} & \multicolumn{3}{c}{ f3 } \\
\cline { 2 - 10 } & Gaus. & Stab. & NIG & Gaus. & Stab. & NIG & Gaus. & Stab. & NIG \\
\hline$D$ & 0.13 & 0.04 & 0.04 & 0.19 & 0.11 & 0.12 & 0.20 & 0.04 & 0.04 \\
$V$ & 0.26 & 0.07 & 0.08 & 0.37 & 0.23 & 0.23 & 0.38 & 0.06 & 0.08 \\
$W^{2}$ & 4.69 & 0.20 & 0.18 & 8.64 & 1.80 & 2.20 & 12.86 & 0.17 & 0.20 \\
$A^{2}$ & 25.21 & 1.37 & 0.97 & 48.89 & 8.68 & 12.99 & 69.92 & 1.03 & 1.35 \\
\hline
\end{tabular}

Table 5: The results of the statistical tests related to the distribution fitting for the emission source AL,T2. The grey shaded cells corresponds the lowest value of test statistic computed for three considering distributions.

- fraction f1 - DOAS $\alpha=1.95$, NIG: $\mu=0, \alpha=38.37, \beta=-0.19, \sigma=0$;

- fraction $\mathrm{f} 2$ - Stable with $\alpha=1.45$, Stable: $\mu=0, \alpha=1.45, \beta=0.01, \sigma=0.001$;

- fraction $\mathrm{f} 3$ - Stable with $\alpha=1.3$, Stable: $\mu=-0.001, \alpha=1.31, \beta=-0.12, \sigma=0.005$.

For each PM fraction we quoted the identified category of distribution and the proposed fitted distribution with its parameters.

\subsection{Emission source FC,T3.}

In this section we consider the statistical description of concentration increments for the PM emitted from forged carbon steel machining using universal machine. This source is indicated by the symbol: FC,T3. 

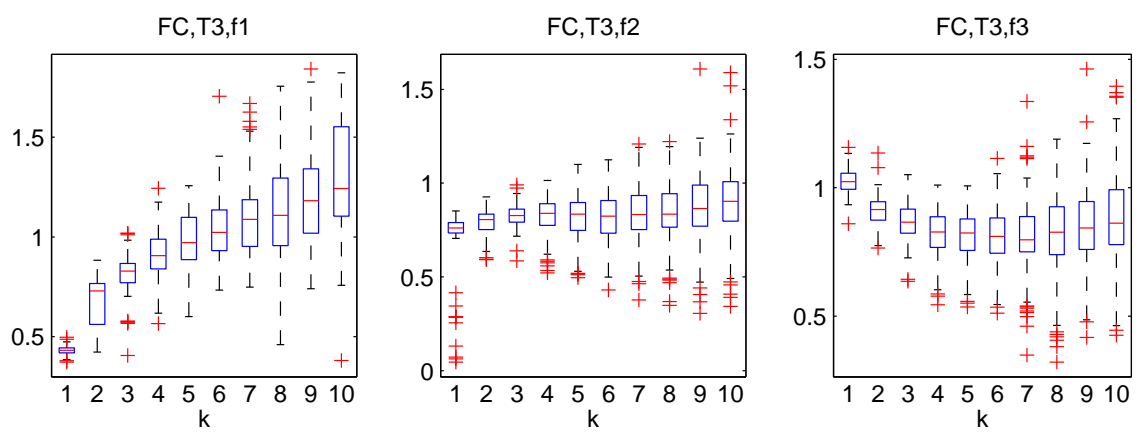

Figure 4: Emission source FC,T3 for three PM fractions f1, f2 and f3; Time series of PM concentration increments (top panels) and the corresponding results of the visual test (bottom panels).

In Fig. 4 (top panels) we display the differenced time series of PM concentrations recorded in the vicinity of FC,T3 emission source for three PM fractions f1, f2 and f3. In Fig. 4 (bottom panel) there are presented the corresponding results of the visual test, which allows to recognize domain of attraction for the distribution in case of each fraction. The summary of recognition is given in Table 2 .

Based on results of visual test, the distribution describing variation of concentration of PM fraction $\mathrm{f} 1$ emitted from source FC,T3 belongs to the domain of attraction of stable distribution. In case of fractions $\mathrm{f} 2$ and $\mathrm{f} 3$, distributions follow stable law. Moreover, $\alpha$ parameters are smaller than 1 and very similar.

In Table 6 there are shown the results of statistical tests regarding a particular distribution fitting for PM fractions f1, f2 and f3 emitted from source AL,T1. We present the values of all test statistics applied.

Two of three results of fitting are consistent with Table 2, i.e. the time series f2 is suspected to come from pure stable distribution, and the fraction $\mathrm{f} 3$ is suspected to be NIG distributed from domain of attraction of stable law. The only exception and small deviation from results of Table 2 is the time series related to the data f1. But in such case the values of the test statistics are very close and difficult to clearly distinguish between NIG (or other distribution from domain of attraction of stable law) and pure stable distribution, see Table 6 for f1. Additionally we present the plot of the right empirical tail with fitted tails of proposed distribution in Fig. 5. From Fig. 5 we see that series $\mathrm{f} 1$ is definitely not stable distributed, 


\begin{tabular}{c|ccc|ccc|ccc}
\multirow{2}{*}{ FC } & \multicolumn{3}{|c|}{$\mathrm{f} 1$} & \multicolumn{3}{c|}{$\mathrm{f} 2$} & \multicolumn{3}{c}{$\mathrm{f} 3$} \\
\cline { 2 - 10 } & Gaus. & Stab. & NIG & Gaus. & Stab. & NIG & Gaus. & Stab. & NIG \\
\hline$D$ & 0.27 & 0.06 & 0.07 & 0.31 & 0.12 & 0.13 & 0.33 & 0.07 & 0.06 \\
$V$ & 0.53 & 0.12 & 0.15 & 0.62 & 0.24 & 0.24 & 0.65 & 0.11 & 0.11 \\
$W^{2}$ & 26.51 & 0.62 & 1.33 & 33.69 & 1.33 & 1.50 & 34.11 & 0.44 & 0.41 \\
$A^{2}$ & 128.12 & 8.15 & 10.34 & 162.14 & 5.90 & 8.04 & 163.32 & 2.44 & 2.25 \\
\hline
\end{tabular}

Table 6: The results of the statistical tests related to the distribution fitting for the emission source FC,T3. The grey shaded cells corresponds the lowest value of test statistic computed for three considering distributions.
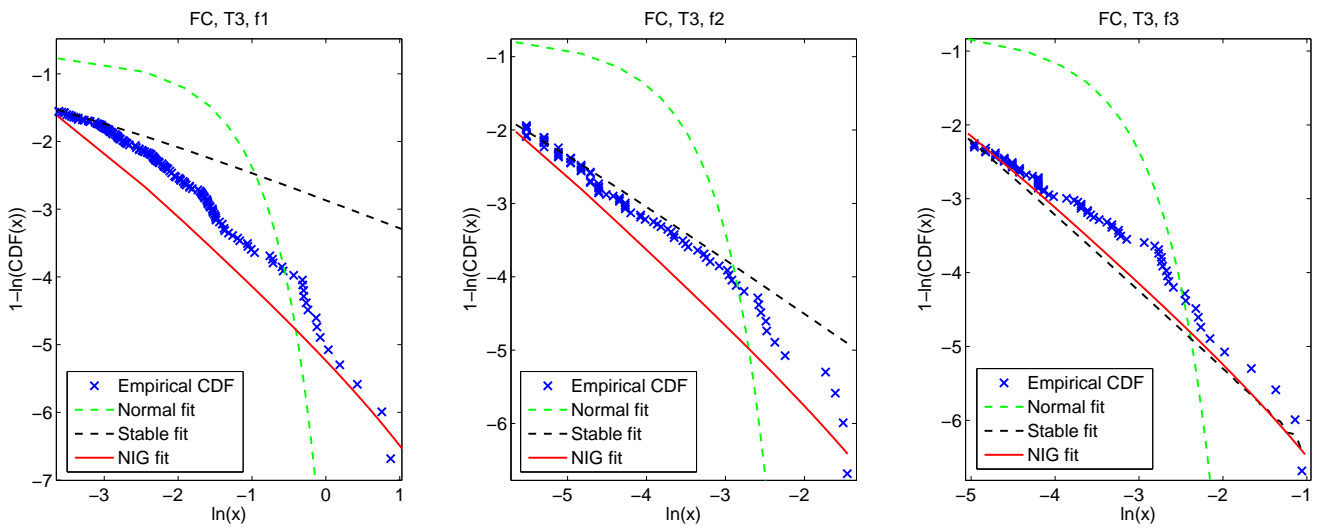

Figure 5: The empirical right tails and fitted tails corresponding the proposed distributions for emission source FC,T3.

which is in contrast with Table 6. Extreme values of series f1 seem to come from NIG distribution, but the middle part of data are between pure stable and NIG law. The series f2 is similar to the stable cdf, especially the middle part of data. Only few extreme big outliers are located between stable and NIG cdf. Finally the series f3 seems to be closer to NIG then to the pure stable distribution. Therefore we can conclude that $\mathrm{f} 1$ and $\mathrm{f} 2$ series are NIG distributed and $\mathrm{f} 2$ comes from stable law. The parameters of fitted distributions we present in Table 4.

In summary, the statistical description of concentration increments for the PM emitted from forged carbon steel machining using universal machine (FC,T3) was following:

- fraction f1 - DOAS $\alpha=1.25$, NIG: $\mu=0, \alpha=0.11, \beta=0.004, \sigma=0.01$;

- fraction $\mathrm{f} 2$ - Stable with $\alpha=0.75$, Stable: $\mu=0, \alpha=0.75, \beta=-0.05, \sigma=0.001$;

- fraction f3 - DOAS $\alpha=0.8, \mathrm{NIG}: \mu=0, \alpha=0.77, \beta=0.01, \sigma=0.002$.

For each PM fraction we quoted the identified category of distribution and the proposed fitted distribution with its parameters.

\subsection{Emission source FC,T4.}

In this section we consider the statistical description of concentration increments for the PM emitted from forged carbon steel welding using ordinary welding. This source is 

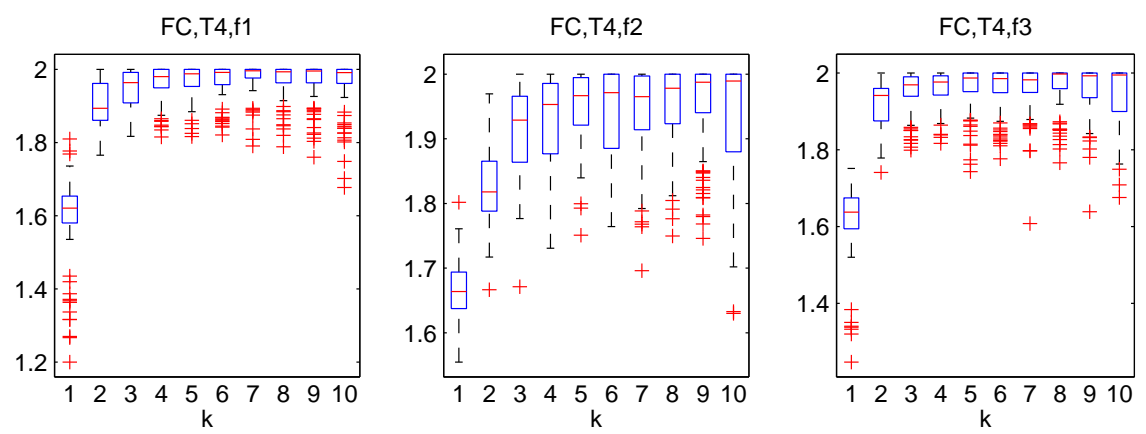

Figure 6: Emission source FC,T4 for three PM fractions f1, f2 and f3; Time series of PM concentration increments (top panels) and the corresponding results of the visual test (bottom panels).

indicated by the symbol: FC,T4.

In Fig. 6 (top panels) we display the differenced time series of PM concentrations recorded in the vicinity of FC,T4 emission source for three PM fractions f1, f2 and f3. In Fig. 6 (bottom panel) there are presented the corresponding results of the visual test, which allows to recognize domain of attraction for the distribution in case of each fraction. The summary of recognition is given in Table 2 .

Based on results of visual test, the distributions describing the variation of PM concentration belong to the domain of attraction of stable distribution with $\alpha$ equal 2 for all considered PM fractions, emitted from source FC,T3.

In Table 7 there are shown the results of statistical tests regarding a particular distribution fitting for PM fractions f1, f2 and f3 emitted from source AL,T1. We present the values of all test statistics applied.

As shown in Table 7, for fraction $\mathrm{f} 1$ and $\mathrm{f} 3$ the data seem to be NIG distributed from

\begin{tabular}{c|ccc|ccc|ccc}
\multirow{2}{*}{ FC } & \multicolumn{3}{|c|}{$\mathrm{f} 1$} & \multicolumn{3}{c|}{$\mathrm{f} 2$} & \multicolumn{3}{c}{$\mathrm{f} 3$} \\
\cline { 2 - 10 } & Gaus. & Stab. & NIG & Gaus. & Stab. & NIG & Gaus. & Stab. & NIG \\
\hline$D$ & 0.07 & 0.04 & 0.04 & 0.16 & 0.14 & 0.15 & 0.08 & 0.04 & 0.04 \\
$V$ & 0.15 & 0.08 & 0.07 & 0.31 & 0.28 & 0.30 & 0.15 & 0.08 & 0.08 \\
$W^{2}$ & 1.40 & 0.22 & 0.17 & 3.77 & 3.30 & 3.67 & 1.39 & 0.29 & 0.18 \\
$A^{2}$ & 7.74 & 2.05 & 1.18 & 20.07 & 16.75 & 21.50 & 7.66 & 2.29 & 1.23 \\
\hline
\end{tabular}

Table 7: The results of the statistical tests related to the distribution fitting for the emission source FC,T4. The grey shaded cells corresponds the lowest value of test statistic computed for three considering distributions. 

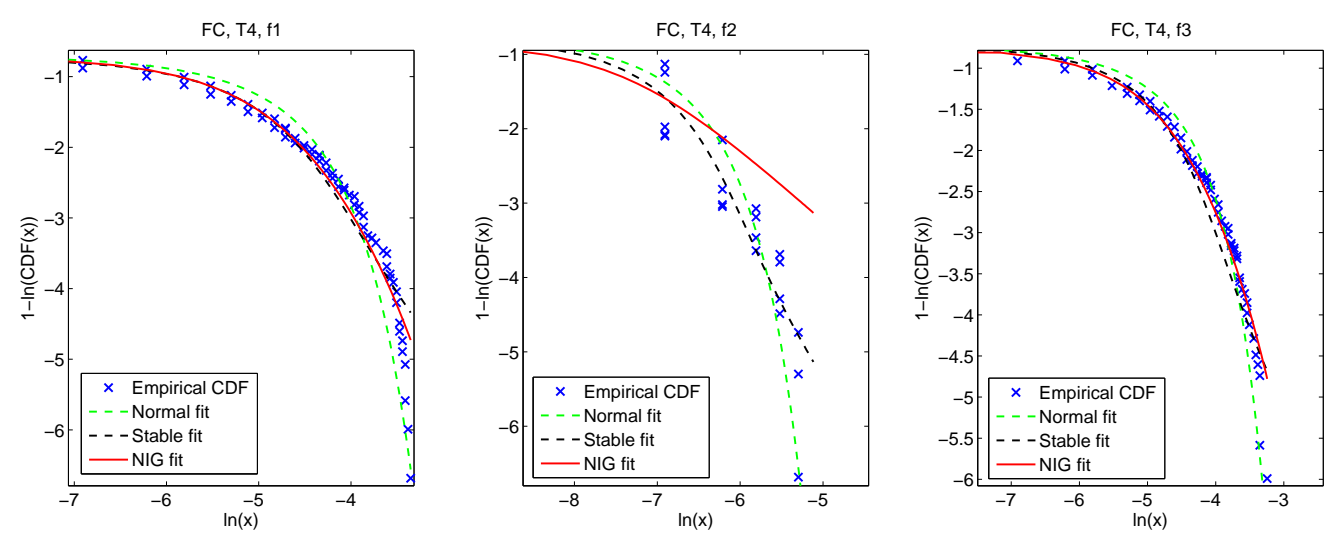

Figure 7: The empirical right tails and fitted tails corresponding the proposed distributions for emission source FC,T4.

domain of attraction of stable law with $\alpha=2$,. Values of test statistics are very close for three proposed distributions. This is consistent to the Table 2 and clearly visible from Fig. 7 , where all tails are close to each other. The only exception from results from Table 2 is the time series related to the data f2. But in such case also the values of the test statistics are very close and difficult to clearly distinguish between NIG (or other distribution from domain of attraction of stable law), pure stable distribution and pure Gaussian. Moreover, from Fig. 7 we see that data of fraction $\mathrm{f} 2$ follow both Gaussian and stable cdfs and not NIG cdf, which indicates the domain of stable law with $\alpha \approx 2$. Hence we generally conclude that all series $\mathrm{f} 1, \mathrm{f} 2$, $\mathrm{f} 3$ comes from domain of attraction of stable law with $\alpha \approx 2$, and they are very close to Gaussian law. Therefore in Table 4 we put the parameters of fitted Gaussian distributions.

In summary, the statistical description of concentration increments for the PM emitted from forged carbon steel welding using ordinary welding (FC,T4) was following:

- fraction f1 - DOAS $\alpha=2$, Gauss: $\mu=0, \sigma=0.011$;

- fraction $\mathrm{f} 2-$ DOAS $\alpha=2$, Gauss: $\mu=0, \alpha=0.001$;

- fraction f3 - DOAS $\alpha=2$, Gauss: $\mu=0, \alpha=0.013$.

For each PM fraction we quoted the identified category of distribution and the proposed fitted distribution with its parameters.

\subsection{Emission source PL,T2}

In this section we consider the statistical description of concentration increments for the PM emitted from plastic machining using Monoblock machine. This source is indicated by the symbol: Pl,T2.

In Fig. 8 (top panels) we display the differenced time series of PM concentrations recorded in the vicinity of PL,T2 emission source for three PM fractions f1, f2 and f3. 

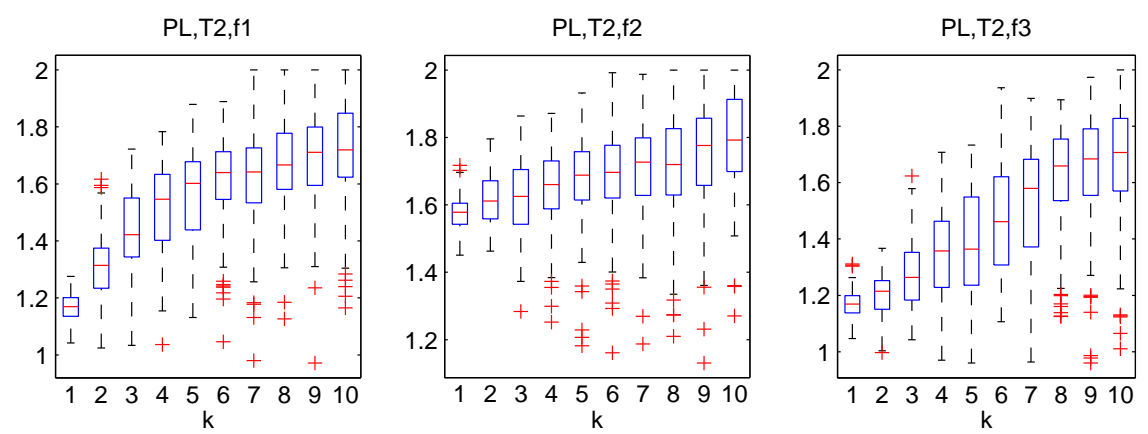

Figure 8: Emission source PL,T2 for three PM fractions f1, f2 and f3; Time series of PM concentration increments (top panels) and the corresponding results of the visual test (bottom panels).

In Fig. 8 (bottom panel) there are presented the corresponding results of the visual test, which allows to recognize domain of attraction for the distribution in case of each fraction. The summary of recognition is given in Table 2 .

Based on results of visual test, the distribution describing variation of concentration of PM fraction f1 emitted from source PL,T2 belongs to the domain of attraction of stable distribution. In case of fractions $\mathrm{f} 2$ and $\mathrm{f} 3$, distributions follow stable law.

In Table 8 there are shown the results of statistical tests regarding a particular distribution fitting for PM fractions f1, f2 and f3 emitted from source AL,T1. We present the values of all test statistics applied.

\begin{tabular}{c|ccc|ccc|ccc}
\multirow{2}{*}{$\mathrm{PL}$} & \multicolumn{3}{|c|}{$\mathrm{f1}$} & \multicolumn{3}{c|}{$\mathrm{f} 2$} & \multicolumn{3}{c}{$\mathrm{f} 3$} \\
\cline { 2 - 11 } & Gaus. & Stab. & NIG & Gaus. & Stab. & NIG & Gaus. & Stab. & NIG \\
\hline$D$ & 0.20 & 0.08 & 0.07 & 0.19 & 0.19 & 0.20 & 0.19 & 0.03 & 0.04 \\
$V$ & 0.38 & 0.15 & 0.15 & 0.39 & 0.39 & 0.39 & 0.39 & 0.06 & 0.06 \\
$W^{2}$ & 10.16 & 0.80 & 0.66 & 8.07 & 5.91 & 6.21 & 12.25 & 0.10 & 0.22 \\
$A^{2}$ & 53.34 & 4.45 & 3.11 & 42.46 & 29.28 & 32.63 & 64.50 & 0.58 & 1.71 \\
\hline
\end{tabular}

Table 8: The results of the statistical tests related to the distribution fitting for the emission source PL,T2. The grey shaded cells corresponds the lowest value of test statistic computed for three considering distributions. 
All results of distribution fitting are strongly consistent with the Table 2. More precisely, only fraction $\mathrm{f} 1$ seems to be NIG distributed from domain of attraction of stable law and the other f2, f3 fractions come from pure stable distribution. The parameters of fitted distributions we present in Table 4.

In summary, the statistical description of concentration increments for the PM emitted from plastic machining using Monoblock machine (P1,T2) was following:

- fraction f1 - DOAS $\alpha=1.6$, NIG: $\mu=0, \alpha=42.45, \beta=-0.22, \sigma=0.002$;

- fraction $\mathrm{f} 2$ - Stable with $\alpha=1.8$, Stable: $\mu=0, \alpha=1.56, \beta=-0.22, \sigma=0.001$;

- fraction $\mathrm{f} 3$ - Stable with $\alpha=1.6$, Stable: $\mu=0, \alpha=1.18, \beta=-0.025, \sigma=0.005$.

For each PM fraction we quoted the identified category of distribution and the proposed fitted distribution with its parameters.

\subsection{Emission source AL,T5}

In this section we consider the statistical description of concentration increments for the PM emitted from aluminum welding using Telwin welder superior. This source is indicated by the symbol: Al,T5.

In Fig. 9 (top panels) we display the differenced time series of PM concentrations recorded in the vicinity of AL,T5 emission source for three PM fractions f1, f2 and f3. In Fig. 9 (bottom panel) there are presented the corresponding results of the visual test, which allows to recognize domain of attraction for the distribution in case of each fraction. The summary of recognition is given in Table 2 .

Based on results of visual test, the distributions describing the variation of PM concentration belong to the domain of attraction of stable distribution with $\alpha$ equal 2 for all considered PM fractions, emitted from source AL,T5.

In Table 9 there are shown the results of statistical tests regarding a particular distribution fitting for PM fractions f1, f2 and f3 emitted from source AL,T1. We present the values of all test statistics applied.

\begin{tabular}{c|ccc|ccc|ccc}
\multirow{2}{*}{$\mathrm{AL}$} & \multicolumn{3}{|c|}{$\mathrm{f} 1$} & \multicolumn{3}{c}{$\mathrm{f} 2$} & \multicolumn{3}{c}{ f3 } \\
\cline { 2 - 10 } & Gaus. & Stab. & NIG & Gaus. & Stab. & NIG & Gaus. & Stab. & NIG \\
\hline$D$ & 0.16 & 0.09 & 0.10 & 0.24 & 0.24 & 0.25 & 0.20 & 0.20 & 0.20 \\
$V$ & 0.31 & 0.17 & 0.18 & 0.48 & 0.48 & 0.48 & 0.40 & 0.40 & 0.40 \\
$W^{2}$ & 5.47 & 1.01 & 1.19 & 9.41 & 9.42 & 12.23 & 6.30 & 5.88 & 5.95 \\
$A^{2}$ & 30.06 & 5.26 & 6.89 & 46.17 & 46.01 & 84.98 & 31.87 & 28.66 & 29.02 \\
\hline
\end{tabular}

Table 9: The results of the statistical tests related to the distribution fitting for the emission source AL,T5. The grey shaded cells corresponds the lowest value of test statistic computed for three considering distributions.

The series corresponding the f1 fraction seems to be stable distributed, but the values of test statistics are also law for NIG distribution. The fractions $\mathrm{f} 2, \mathrm{f} 3$ have law values for each of proposed distributions, which indicate the data distribution is from domain of attraction 

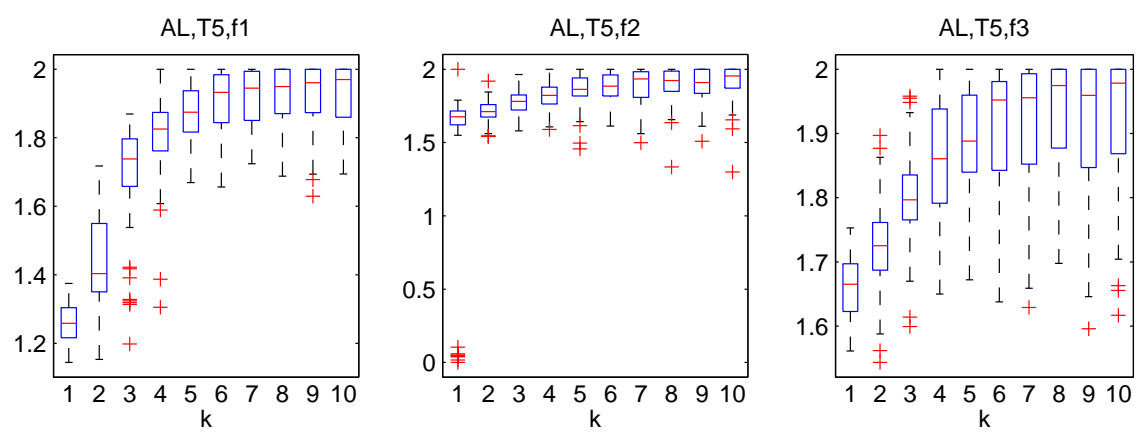

Figure 9: Emission source AL,T5 for three PM fractions f1, f2 and f3; Time series of PM concentration increments (top panels) and the corresponding results of the visual test (bottom panels).

of stable law with $\alpha$ parameter close to 2. Also from Fig. 10 we can conclude that right empirical tails of data are close to stable as well as close to Gaussian and NIG distribution. There is no enough statistical evidence to reject or accept the hypothesis of some specified distribution. Therefore in final Table 4 we state that the distribution of AL, T5 series is difficult to identify.

In summary, the statistical description of concentration increments for the PM emitted from aluminium welding using Telwin welder superior (Al,T5) was following:

- fraction $\mathrm{f} 1$ - DOAS $\alpha=1.95$, not identified;

- fraction $\mathrm{f} 2-$ DOAS $\alpha=1.95$, not identified;

- fraction f3 - DOAS $\alpha=1.95$, not identified.

For each PM fraction we quoted the identified category of distribution and indicated the inability to fit a particular distribution.

\section{Discussion and conclusions}

In this work we studied the method which could be applied to discriminate between various PM emission sources. The approach is based on two elements: measurements of PM concentration in the air and stochastic analysis of the obtained results. The goal of measurements is to provide data on the temporal variation of PM concentration in the 

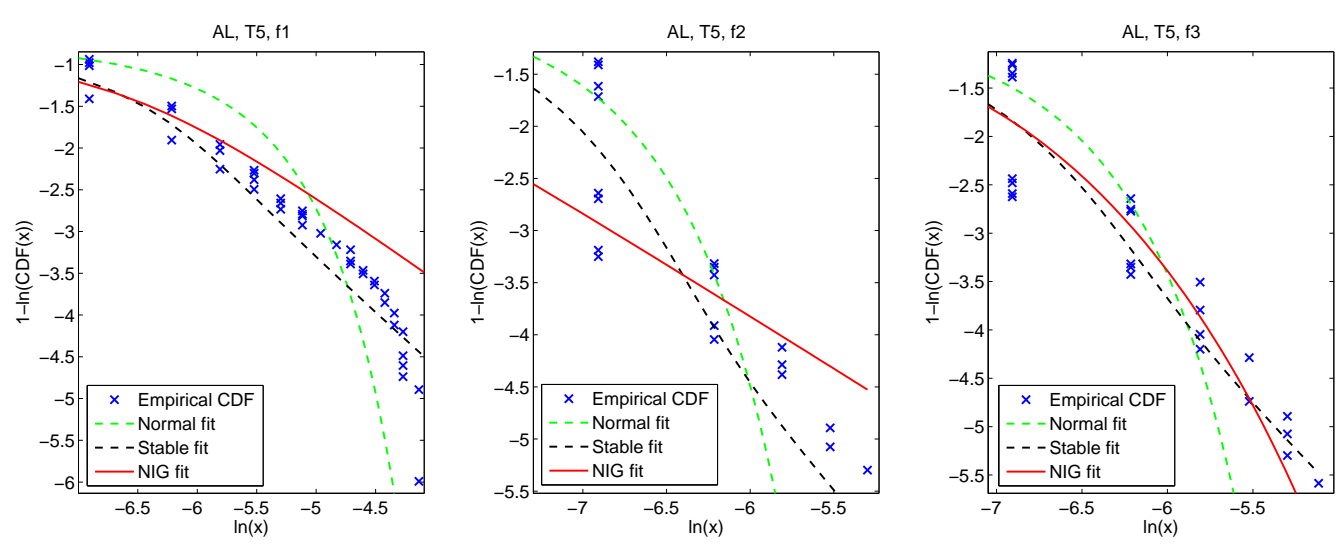

Figure 10: The empirical right tails and fitted tails corresponding the proposed distributions for emission source AL,T5.

vicinity of emission source. Hence, they shall be performed continuously and in the period of time which is sufficient to account for the characteristic behavior of the source. The reason for applying stochastic methods is to study PM concentration variation. In this work, the differenced time series of this parameter were directly examined. The stochastic analysis was performed in two steps. The first one consisted in recognizing the category of distribution for the data. At this stage we distinguished between stable and the domain of attraction of stable distribution. The second step involved distribution fitting. There were considered three possible probability laws underlying the time series: Gaussian, stable, and NIG.

As shown by the obtained results, PM emission sources could be distinguished based on the statistical distribution of PM concentration variations. In principle, the distinction was achieved by recognizing the category of matching distribution. However, the information acquired in the second step of stochastic analysis helped to verify and clarify the results of the first step.

The studied discriminative approach had a number of interesting features. Firstly, it was sensitive to the influence of the kind of material processing (machining, welding) on PM concentration variation. We observed that increments of PM concentration associated with machining (sources: Al,T1, Al,T2, FCT3 and PL,T2) followed either stable or NIG distribution. On the other hand, in case of welding (sources: FC,T4 and Al,T5) Gaussian distribution fitted best or the actual distribution could not be found, see Table 2 and Table 4 . Secondly, with the considered approach it was possible to discriminate sources which differed due to the machine/tool applied for material processing. The relevant example is aluminum machining with the use of Hass machine (Al,T1) and applying Monoblock machine (Al,T2). As shown in Table 4 dust fractions $\mathrm{f} 2$ and $\mathrm{f} 3$ emitted from the first source were characterized by NIG distribution, and from the second source - by the stable distribution. Thirdly, the presented discriminative method was weakly sensitive to differences between sources due to the material processed. The illustrative example is to compare machining of aluminum $(\mathrm{Al}, \mathrm{T} 2)$ and machining of plastic $(\mathrm{Pl}, \mathrm{T} 2)$ with the same machine. As shown in Table 4 
nearly identical distributions were fitted to the corresponding PM fractions: f1, f2 and f3 emitted from the two sources. We may suppose that for fine particles, as considered in this work i.e. less or equal to $4 \mu \mathrm{m}$ diameter, the material does not matter for the temporal variation of PM concentration but the process of dust formation is decisive for the ability to discriminate between sources.

However, the access to information on size-segregated mass fraction concentrations was crucial for achieving high discriminative potential of the method. Considering three fractions in the analysis introduced the possibility to describe each source by three complex descriptors (type of probability distribution and in most cases the parameters of fitted distribution). Typically, distribution matching fraction $\mathrm{f} 1$ was different than those fitted for fractions $\mathrm{f} 2$ and f3, all dust emitted from the same source. In case of the last two fractions, although the kind of distribution was usually the same, the differences in parameters were observed.

Technically, the method is robust regarding the magnitude of PM concentration because. In the analysis there are utilized concentration changes. However, temporal variation of PM concentration in the air may be dependent on concentration magnitude due to interactions between particles, in particular when the PM concentration is high. However, from the perspective of the problem solved, we would consider such influence as indicative for the source behavior and contributing to its distinctive character. The PM concentration we measured was in wide range from $0.1 \mathrm{mg} / \mathrm{m}^{3}$ to $100 \mathrm{mg} / \mathrm{m}^{3}$. Hence the existence and potential contribution of the mentioned effect may not be excluded.

It shall be underlined, that the presented results were achieved when applying one measurement method (even one measuring instrument) to collect data about PM concentration. We have not been considering changes in the discrimination performance due to the PM measurement method applied. The interest was in evaluating the entire approach in view of the PM characteristics which may be acquired using a particular method of measurement. The one we chose is relatively affordable and may be considered as an option in practical applications of this kind.

In our opinion, the presented approach is very promising and it deserves further study and development.

\section{Acknowledgment}

The work was supported by the National Science Center, Poland, under the contract number DEC-2012/07/B/ST8/03031.

\section{References}

[1] H. Knudsen \& N. Rasmussen(ed.). Particulate Matter: Sources, Emission Rates and Health Effects. Environmental Science, Engineering and Technology. Pollution Science, Technology and Abatement (2012).

[2] J. Xi, J.W. Kim, X.A. Si, Y. Zhou. Diagnosing obstructive respiratory diseases using exhaled aerosol fingerprints: A feasibility study. Journal of Aerosol Science, 64, 24-36 (2013).

[3] R.H.K. Entink, C. Bekker , W.F. Fransman, D.H. Brouwer Analysis of time series of particle size distributions in nano exposure assessment. Journal of Aerosol Science, 81, 62-69 (2015). 
[4] B. Hołyńska, J. Ptasiński, W. Maenhaut, H.J. Annegarn. Energy-dispersive X-ray fluorescence spectrometer with capillary optics for the chemical analysis of atmospheric aerosols with high time resolution. Journal of Aerosol Science, 28(8), 1455-1463 (1997).

[5] A. Hess, M. Tarik, Ch. Ludwig. A hyphenated SMPS-ICPMS coupling setup: Size-resolved element specific analysis of airborne nanoparticles. Journal of Aerosol Science, 88, 109-118 (2015).

[6] A. Arffman, J. Yli-Ojanperä, J. Kalliokoski, J. Harra, L. Pirjola, P. Karjalainen, T. Rönkkö, J. Keskinen. High-resolution low-pressure cascade impactor. Journal of Aerosol Science, 78, 97-109 (2014).

[7] M.N.A. Karlsson, G. Frank, B.G. Martinsson. Measurement of the differential mobility analyser transfer function. 31(1), 23-24 (2000).

[8] X. Wang, R. Caldow, G.J. Sem, N. Hama, H. Sakurai. Evaluation of a condensation particle counter for vehicle emission measurement: Experimental procedure and effects of calibration aerosol material, Journal of Aerosol Science, 41(3) 306-318 (2010).

[9] M. He., S. Dhaniyala. Comparison of diffusion charging and mobility-based methods for measurement of aerosol agglomerate surface area. Journal of Aerosol Science, 47, 100-110 (2012).

[10] E. Ruppecht, M. Meyer, H. Patashnick. The tapered element oscillating microbalance as a tool for measuring ambient particulate concentrations in real time. Journal of Aerosol Science, 23(1), 635-638 (1992).

[11] J.H. Lee, P.K. Hopke, T.M. Holsen, D.-W. Lee, P.A. Jaques, C. Sioutas, J.L. Ambs. Performance eval

[12] R. Khettabi, V. Songmene, J. Masounave. Influence of machining processes on particles emission. 49th Annual Conf. of Metallurgists of CIM, Vancouver, 277-288 (2010c).

[13] V. Songmene, B. Balout, J. Masounave. Clean Machining: Experimental Investigation on Dust Formation - Part I: Influence of Machining Parameters and Chip Formation. Int. J. of Environmentally Conscious Design and Manufacturing, 14(1): 1-16 (2008a).

[14] V. Songmene, B. Balout, J. Masounave. Clean Machining: Experimental Investigation on Dust Formation - Part II: Influence of Machining Strategies and Drill Condition. Int. J. of Environmentally Conscious Design and Manufacturing, 14(1): 17- 33 (2008b).

[15] N.T. Jenkins. Chemistry of Air- borne Particles from Metallurgical Processing. Ph.D. dissertation. Cambridge, Mass., Massachusetts Institute of Technology (2003).

[16] A.P. Malshe, M.A. Taher, et all. A Comparative Study of Dry Machining of A390 Alloy using PCD and CVD Diamond Tools, Trans. NAMRI/SME Vol. XXVI: 267-272 (1998).

[17] V. Songmene, R. Khettabi, I. Zaghbani, J. Kouam, \& A. Djebara Machining and Machinability of Aluminum Alloys, www.intechopen.com, 377-400 (2012).

[18] D.D. Bell, J. Chou, L. Nowag \& S.Y. Liang. Modeling of the Environmental Effect of Cutting Fluid. Tribology transactions, 42(1), 168-173 (1999).

[19] R. Khettabi, V. Songmene. Modeling of Particle Emission during Dry Orthogonal Cutting. Journal of Materials Engineering and Performance. 19(6), p. 776-789 (2010).uation of continuous PM2.5 mass concentration monitors. Journal of Aerosol Science, 36(1), 95-109 (2005).

[20] J.P. Nolan. Fitting Data and Assessing Goodness-of-fit with Stable Distributions. Washington, DC 20016 USA (1999).

[21] R. Weron. On the Chambers-Mallows-Stuck method for simulating skewed stable random variables. Statistics \& Probability Letters, 28, 165-171 (1996).

[22] G. Żak, A. Wyłomańska, R. Zimroz. Application of alpha-stable distribution approach for local damage detection in rotating machines, Journal of Vibroengineering 17(6), 2987-3002 (2015).

[23] J.P. Nolan. Stable Distribution: Models for Heavy Tailed Data (2014).

[24] H. Fofack \& J. P. Nolan. Tail Behavior, Modes and Other Characteristics of Stable Distributions (2000).

[25] B.V. Gnedenko \& A.N. Kolmogorov. Limit Distributions of Sums of Independent Random Variables. Addison-Wesley, Cambridge (1954).

[26] K. Burnecki, A. Wyłomańska, A. Chechkin. Discriminating between light- and heavy-tailed distributions with limit theorem, PLoS ONE 10(12) (2015).

[27] G. Samorodnitsky, M.S. Taqqu Stable Non-Gaussian Random Processes, Chapman \& Hall, New York (1994). 
[28] R. Kuske, J. B. Keller. Rate of convergence to a stable law. SIAM J. Appl. Math. 61: 1308-1323 (2000).

[29] I.A. Koutrouvelis. Regression-type estimation of the parameters of stable laws. J. Amer. Statist. Assoc. 75: 918-928 (1980).

[30] I.A. Koutrouvelis. An interative procedure for the estimation of the parameters of stable laws. Commun. Statist. Simul. Comput. 10: 17-28 (1981).

[31] R. Weron. Computationally intensive value at risk calculations. In J. Gentle, W. Härdle, \& Y. Mori, editors, Handbook of Computational Statistics, 911, Springer, Berlin (2004).

[32] Y. Benjamini Opening the Box of a Boxplot. Amer Statist. 42, 257-262 (1988).

[33] A.C. Davison, D.V. Hinkley. Bootstrap Methods and their Application. Cambridge Series in Statistical and Probabilistic Mathematics. Cambridge: Cambridge University Press (1999).

[34] O.E. Barndorff-Nielsen Normal inverse Gaussian Processes and the Modelling of Stock Returns. Research Report 300, Department of Theoretical Statistics, University of Aarhus (1995).

[35] O.E. Barndorff-Nielsen, T. Mikosch \& S.I. Resnick Lévy Processes: Theory and Applications. Birkhäuser (2013). 